\title{
On Starshaped Intuitionistic Fuzzy Sets
}

\author{
Weihua Xu ${ }^{1,2}$, Yufeng Liu ${ }^{1}$, Wenxin Sun ${ }^{1}$ \\ ${ }^{1}$ The Mathematics and Statistics, Chongqing University of Technology, Chongqing, China \\ ${ }^{2}$ School of Management, Xi' an Jiaotong University, Xi'an, China \\ E-mail:chxuwh@gmail.com, liuyufeng@cqut.edu.cn, sunxuxin520@163.com \\ Received May 23, 2011; revised June 28, 2011; accepted July 6, 2011
}

\begin{abstract}
Intuitionistic fuzzy starshaped sets (i.f.s.) is a generalized model of fuzzy starshaped set. By the definition of i.f.s., the intuitionistic fuzzy general starshaped sets (i.f.g.s.), intuitionistic fuzzy quasi-starshaped sets (i.f.q-s.) and intuitionistic fuzzy pseudo-starshaped sets (i.f.p-s.) are proposed and the relationships among them are studied. The equivalent discrimination conditions of i.f.q-s. and i.f.p-s. are presented on the basis of their properties which are meaningful for the research of the generalized fuzzy starshaped sets. Moreover, the invariance of the two given fuzzy sets under the translation transformation and linear reversible transformation are discussed.
\end{abstract}

Keywords: Intuitionistic Fuzzy Starshaped Sets, Cut Sets, Starshapedness, Convex Sets

\section{Introduction}

Since the theory of fuzzy set was proposed by Zadeh in 1965 , it has been widely used such as fuzzy control, fuzzy recognition, fuzzy evaluation, system theory, information retrieval and so on. The intuitionistic fuzzy set theory, proposed by Atanassov [1], is an extension of the fuzzy set theory. The core idea of intuitionistic fuzzy sets is added a non-membership functions in the basis of membership functions, which can cooperate with the membership function and describe the fuzzy object of the world more exquisitely. At present, the research of intuition fuzzy set theory is more active in international, especially the research of some similar problems in fuzzy set. Through more than 20 years of development, the intuitionistic fuzzy set has made a lot of important achievements [2-9].

With the deepening of the research work and expansion, another more worthy of concern is the emergence of fuzzy starshaped set theory [10] and its applications. Fuzzy starshaped sets have more abundant properties and characteristics, it is a directly extension of the fuzzy set theory and convex set, many useful results have been obtained.

In this paper, we define a new kind of fuzzy set which is intuition fuzzy set, by combining the fuzzy starshaped set and intuition fuzzy set. By the basic definition of intuitionistic fuzzy starshaped set, we introduce some new different types of starshapedness. We discuss the relationships among these different types of starshapedness, and obtain some important properties.

\section{Preliminaries}

Let $x, y \in R^{n}, \overline{x y}=\{z \mid z=\alpha x+\beta y\}$ is a line segment, where $\alpha \geq 0, \beta \geq 0, \alpha+\beta=1$. A set $S$ is simply said to be starshaped relative to a point $x \in R^{n}$, if $\overline{x y} \subseteq S$ for any point $y \in S$. A set $S$ is simply said to be starshaped, which means that there exists a point $x$ in $R^{n}$ such that $S$ is starshaped relative to $x$. The kernel ker $S$ of $S$ is the set of all points $x \in S$ such that $\overline{x y} \subseteq S$ for any $y \in S$.

Definition 2.1. Let $R^{n}$ denote an universe of discourse. An intuitionistic fuzzy set $\widetilde{A}$ is an object having the form

$$
\widetilde{A}=\left\{\left(x, \mu_{\tilde{A}}(x), v_{\widetilde{A}}(x) \mid x \in R^{n}\right)\right\}
$$

where $\mu_{\tilde{A}}: R^{n} \rightarrow[0,1], v_{\tilde{A}}: R^{n} \rightarrow[0,1]$ satisfy $0 \leq \mu_{\tilde{A}}+v_{\tilde{A}} \leq 1$ for all $x \in R^{n}, \mu_{\tilde{A}}$ and $v_{\tilde{A}}$ are called the degree of membership and the one degree of nonmembership of the element $x$ to $\widetilde{A}$ respectively. Let $F\left(R^{n}\right)$ be the classes of normal intuitionistic fuzzy sets on $R^{n}$, that is $\left\{x \in R^{n} \mid \mu_{\tilde{A}}(x)=1, v_{\tilde{A}}(x)=0\right\}$ is nonempty.

Example 2.1. Let $\widetilde{A}=\left\{\left(x, \mu_{\widetilde{A}}(x), v_{\widetilde{A}}(x) \mid x \in R\right)\right\}$, where 


$$
\begin{aligned}
& \mu_{\tilde{A}}(x)= \begin{cases}x+1 & x \in[-1,0] \\
-x+1 & x \in(0,1] \\
0 & \text { otherwise }\end{cases} \\
& v_{\tilde{A}}(x)=\left\{\begin{array}{cc}
-x & x \in[-1,0] \\
x & x \in(0,1] \\
0 & \text { otherwise }
\end{array}\right.
\end{aligned}
$$

Then $\widetilde{A} \in F(R)$.

Definition 2.2. An intuitionistic fuzzy set $\widetilde{A} \in F\left(R^{n}\right)$ is called quasi-convex if

$$
\begin{aligned}
& \mu_{\widetilde{A}}(\lambda(x-y)+y) \geq \min \left\{\mu_{\widetilde{A}}(x), \mu_{\widetilde{A}}(y)\right\} \\
& v_{\widetilde{A}}(\lambda(x-y)+y) \leq \max \left\{v_{\widetilde{A}}(x), v_{\widetilde{A}}(y)\right\}
\end{aligned}
$$

for all $x, y \in R^{n}, \lambda \in[0,1]$

Definition 2.3. Let $\widetilde{A}, \widetilde{B} \in F\left(R^{n}\right)$, the union, intersection and complement of $\widetilde{A}$ and $\widetilde{B}$ are defined as follows,

$$
\begin{aligned}
& \widetilde{A} \cup \widetilde{B}=\left\{\left(x, \mu_{\widetilde{A}}(x) \vee \mu_{\widetilde{B}}(x), v_{\widetilde{A}}(x) \wedge v_{\widetilde{B}}(x)\right) \mid x \in R^{n}\right\} \\
& \widetilde{A} \cup \widetilde{B}=\left\{\left(x, \mu_{\widetilde{A}}(x) \wedge \mu_{\widetilde{B}}(x), v_{\widetilde{A}}(x) \vee v_{\widetilde{B}}(x)\right) \mid x \in R^{n}\right\} \\
& \widetilde{A}^{c}=\left\{\left(x, 1-\mu_{\widetilde{A}}(x), 1-v_{\widetilde{A}}(x)\right) \mid x \in R^{n}\right\}
\end{aligned}
$$

Definition 2.4. [11] Let $\widetilde{A}, \widetilde{B} \in F\left(R^{n}\right), \quad \alpha, \beta \in[0,1]$, the $[\alpha, \beta]-$ cut , $[\alpha, \beta)$-cut, $(\alpha, \beta]-$ cut and $(\alpha, \beta)-$ cut of $A$ are defined as follows:

$$
\begin{aligned}
& \widetilde{A}_{[\alpha, \beta]}=\left\{x \mid x \in X, \mu_{\widetilde{A}}(x) \geq \alpha, v_{\widetilde{A}}(x) \leq \beta\right\} \\
& \widetilde{A}_{[\alpha, \beta)}=\left\{x \mid x \in X, \mu_{\widetilde{A}}(x) \geq \alpha, v_{\widetilde{A}}(x)<\beta\right\} \\
& \widetilde{A}_{(\alpha, \beta]}=\left\{x \mid x \in X, \mu_{\widetilde{A}}(x)>\alpha, v_{\widetilde{A}}(x) \leq \beta\right\} \\
& \widetilde{A}_{(\alpha, \beta)}=\left\{x \mid x \in X, \mu_{\widetilde{A}}(x)>\alpha, v_{\widetilde{A}}(x)<\beta\right\}
\end{aligned}
$$

\section{Starshaped Intuitionistic Fuzzy Sets}

Definition 3.1 An intuitionistic fuzzy set $\widetilde{A} \in F\left(R^{n}\right)$ is said to be i.f.s. relative to $y \in R^{n}$ if

$$
\mu_{\tilde{A}}(\lambda(x-y)+y) \geq \mu_{\widetilde{A}}(x)
$$

and

$$
v_{\widetilde{A}}(\lambda(x-y)+y) \leq \mu_{\tilde{A}}(x)
$$

for all $x \in R^{n}, \lambda \in[0,1]$,

Proposition 3.1 Let $\widetilde{A} \in F\left(R^{n}\right)$ is i.f.s. relative to $y \in R^{n}$, then

$$
\mu_{\widetilde{A}}(y)=\sup _{x \in R^{n}}\left\{\mu_{\widetilde{A}}(x)\right\}=1
$$

$$
v_{\widetilde{A}}(y)=\inf _{x \in R^{n}}\left\{v_{\widetilde{A}}(x)\right\}=0
$$

Proof. Let $\widetilde{A}$ is i.f.s. relative to $y$, then for all $x \in R^{n}$,

$$
\mu_{\widetilde{A}}(\lambda(x-y)+y) \geq \mu_{\widetilde{A}}(x)
$$

and

$$
v_{\widetilde{A}}(\lambda(x-y)+y) \leq v_{\widetilde{A}}(x)
$$

are true for $0 \leq \lambda \leq 1$.Thus, only take $\lambda=0$, it can be found that $\mu_{\widetilde{A}}(y) \geq \mu_{\widetilde{A}}(x)$ and $v_{\widetilde{A}}(y) \leq v_{\widetilde{A}}(x)$ are true for all $x \in R^{n}$.

Hence

$$
\mu_{\widetilde{A}}(y)=\sup _{x \in R^{n}}\left\{\mu_{\widetilde{A}}(x)\right\}=1
$$

and

$$
v_{\widetilde{A}}(y)=\inf _{x \in R^{n}}\left\{v_{\widetilde{A}}(x)\right\}=0
$$

Example 3.1. The intuitionistic fuzzy $\widetilde{A} \in F(R)$ with

$$
\begin{aligned}
& \mu_{\tilde{A}}(x)= \begin{cases}\mathrm{e}^{x} & x \in(-\infty, 0] \\
\mathrm{e}^{-x} & x \in(0,+\infty)\end{cases} \\
& v_{\widetilde{A}}(x)= \begin{cases}1-\mathrm{e}^{x} & x \in(-\infty, 0] \\
1-\mathrm{e}^{-x} & x \in(0,+\infty)\end{cases}
\end{aligned}
$$

is i.f.s. relative to $y=0$.

Proposition 3.2. An intuitionistic fuzzy set $\widetilde{A} \in F\left(R^{n}\right)$ is i.f.s. respect to $y \in R^{n}$ iff its level sets are starshaped relative to $\mathrm{y}$.

Proof. Suppose $\widetilde{A}_{[\alpha, \beta]}$ is starshaped relative to $y \in R^{n}$ for all $\alpha, \beta \in[0,1]$. For $x \in R^{n}$, let $\alpha=\mu_{\widetilde{A}}(x), \beta=v_{\widetilde{A}}(x)$, then $\overline{x y} \in \widetilde{A}_{[\alpha, \beta]}$. That is, for any $\lambda \in[0.1]$

$$
\mu_{\tilde{A}}(\lambda(x-y)+y) \geq \alpha=\mu_{\tilde{A}}(x)
$$

and

$$
v_{\tilde{A}}(\lambda(x-y)+y) \leq \beta=v_{\tilde{A}}(x)
$$

Conversely, if for all $x \in R^{n}, \lambda \in[0,1]$,

$$
\mu_{\widetilde{A}}(\lambda(x-y)+y) \geq \mu_{\widetilde{A}}(x)
$$

and

$$
v_{\widetilde{A}}(\lambda(x-y)+y) \leq v_{\widetilde{A}}(x)
$$

hold. Since $\widetilde{A}_{[\alpha, \beta]} \neq \varnothing$, there exists $x \in \widetilde{A}_{[\alpha, \beta]}$, that means $\mu_{\tilde{A}}(x) \geq \alpha$ and $v_{\tilde{A}}(x) \leq \beta$. Hence,

$$
\mu_{\widetilde{A}}(\lambda(x-y)+y) \geq \mu_{\widetilde{A}}(x) \geq \alpha
$$

and 


$$
v_{\widetilde{A}}(\lambda(x-y)+y) \leq v_{\widetilde{A}}(x) \leq \beta
$$

for any $\lambda \in[0,1]$.

So $\overline{x y} \in \widetilde{A}_{[\alpha, \beta]}$. Thus $\widetilde{A}_{[\alpha, \beta]}$ is starshaped relative to y.

Definition 3.2. An intuitionistic fuzzy set $\widetilde{A} \in F\left(R^{n}\right)$ is said to be i.f.g.s. if all level sets are starshaped sets in $R^{n}$.

Definition 3.3. An intuitionistic fuzzy set $\widetilde{A} \in F\left(R^{n}\right)$ is said to be i.f.q-s. relative to $y \in R^{n}$ if for all $x \in R^{n}, \lambda \in[0,1]$, the following hold,

$$
\begin{gathered}
\mu_{\tilde{A}}(\lambda(x-y)) \geq \min \left\{\mu_{\tilde{A}}(x), \mu_{\tilde{A}}(y)\right\} \\
v_{\widetilde{A}}(\lambda(x-y)+y) \leq \max \left\{v_{\widetilde{A}}(x), v_{\widetilde{A}}(x)\right\}
\end{gathered}
$$

Definition 3.4 An intuitionistic fuzzy set $\widetilde{A} \in F\left(R^{n}\right)$ is said to be i.f.p-s. relative to $y \in R^{n}$ if for all $x \in R^{n}, \lambda \in[0,1]$, the following are true,

$$
\begin{aligned}
& \mu_{\tilde{A}}(\lambda(x-y)+y) \geq \lambda \mu_{\tilde{A}}(x)+(1-\lambda) \mu_{\tilde{A}}(y) \\
& v_{\widetilde{A}}(\lambda(x-y)+y) \leq \lambda v_{\widetilde{A}}(x)+(1-\lambda) v_{\tilde{A}}(y)
\end{aligned}
$$

Definition 3.4. Let $\operatorname{ker}(\widetilde{A})$ (respectively, $q-\operatorname{ker}(\tilde{A})$, $p-\operatorname{ker}(\tilde{A})$ ) be the totality of $y \in R^{n}$ such that $\tilde{A}$ is i.f.s. (respectively, i.f.q-s., i.f.q-s.) relative to $y$.

Definition 3.5. The intuitionistic fuzzy hypograph of $\widetilde{A}$ denoted by $f . h p y(\widetilde{A})$, is defined as

$$
f . h p y(\widetilde{A})=f . h p y(\mu) \cup f . h p y(v)
$$

where

$$
\begin{aligned}
& f \cdot h p y\left(\mu_{\tilde{A}}\right)=\left\{(x, t) \mid x \in R, t \in\left[0, \mu_{\tilde{A}}(x)\right]\right\} \\
& f \cdot h p y\left(v_{\tilde{A}}\right)=\left\{(x, s) \mid x \in R, s \in\left[v_{\tilde{A}}(x), 1\right]\right\}
\end{aligned}
$$

Theorem 3.1. Let $\tilde{A} \in F\left(R^{n}\right)$ is i.f.g.s. and $\exists y \in \bigcap \operatorname{ker} \widetilde{A}_{[\alpha, \beta]}$ iff $\tilde{A} \in F\left(R^{n}\right)$ is i.f.s. relative to $y$.

Proof. “ $\Rightarrow$ ” Since $\widetilde{A} \in F\left(R^{n}\right)$ is i.f.g.s. and $\bigcap_{\alpha, \beta} \operatorname{ker} \widetilde{A}_{[\alpha, \beta]} \neq \varnothing$, that is $\exists y \in \bigcap_{\alpha, \beta} \operatorname{ker} \widetilde{A}_{[\alpha, \beta]}$. Then $\widetilde{A}_{[\alpha, \beta]}$ is starshaped relative to $y$. By Proposition 3.1 we get that $\widetilde{A}$ is i.f.s. relative to $y$.

" $\Leftarrow$ " it follows directly from Definition 3.2 and Proposition 3.1.

Theorem 3.2. Let $\widetilde{A} \in F\left(R^{n}\right)$ is i.f.p-s. relative to $y \in R^{n}$, then it is i.f.q-s. relative to $y$.

Proof. Since for all $x \in R^{n}, \lambda \in[0,1]$, the following hold,

$$
\begin{aligned}
\mu_{\tilde{A}}(\lambda(x-y)+y) & \geq \lambda \mu_{\tilde{A}}(x)+(1-\lambda) \mu_{\tilde{A}}(y) \\
& \geq \min \left\{\mu_{\tilde{A}}(x), \mu_{\tilde{A}}(y)\right\} \\
v_{\widetilde{A}}(\lambda(x-y)+y) & \leq \lambda v_{\tilde{A}}(x)+(1-\lambda) v_{\widetilde{A}}(y) \\
& \leq \max \left\{v_{\widetilde{A}}(x), v_{\tilde{A}}(y)\right\}
\end{aligned}
$$

Thus $\tilde{A}$ is i.f.q-s. relative to $y$.

Remark 3.1. The inverse statements do not hold in general as shown in the following example.

Example 3.3. The intuitionistic fuzzy $\widetilde{A} \in F\left(R^{n}\right)$ with

$$
\begin{aligned}
& \mu_{\tilde{A}}(x)= \begin{cases}2+x & x \in[-2,-1] \\
x^{2} & x \in[-1,1] \\
2-x & x \in[1,2] \\
0 & \text { otherwise }\end{cases} \\
& v_{\widetilde{A}}(x)= \begin{cases}-x-1 & x \in[-2,-1] \\
1-x^{2} & x \in[-1,1] \\
x-1 & x \in[1,2] \\
1 & \text { otherwise }\end{cases}
\end{aligned}
$$

is i.f.q-s. relative to $y=0$. But it is not i.f.p-s. relative to $y=0$.

Theorem 3.3. Let $\widetilde{A} \in F\left(R^{n}\right)$ is i.f.q-s. relative to $y \in R^{n}$, then

$$
\begin{aligned}
& \mu_{\tilde{A}}(y)=\sup _{x \in R^{n}}\left\{\mu_{\tilde{A}}(x)\right\}=1 \\
& v_{\tilde{A}}(y)=\inf _{x \in R^{n}}\left\{v_{\tilde{A}}(x)\right\}=0
\end{aligned}
$$

iff $\widetilde{A} \in F\left(R^{n}\right)$ is i.f.s. relative to $y$.

Proof. " $\Rightarrow$ ” Since $\widetilde{A} \in F\left(R^{n}\right)$ is i.f.q-s. relative to $y \in R^{n}$,

$$
\mu_{\tilde{A}}(y)=\sup _{x \in R^{n}}\left\{\mu_{\tilde{A}}(x)\right\}=1
$$

and

$$
v_{\tilde{A}}(y)=\inf _{x \in R^{n}}\left\{v_{\tilde{A}}(x)\right\}=0
$$

then for all $x \in R^{n}, \lambda \in[0,1]$, we have

$$
\begin{aligned}
& \mu_{\tilde{A}}(\lambda(x-y)+y) \geq \min \left\{\mu_{\tilde{A}}(x), \mu_{\tilde{A}}(y)\right\}=\mu_{\tilde{A}}(x) \\
& v_{\widetilde{A}}(\lambda(x-y)+y) \leq \max \left\{v_{\widetilde{A}}(x), v_{\widetilde{A}}(y)\right\}=v_{\widetilde{A}}(x)
\end{aligned}
$$

Hence $\tilde{A}$ it is i.f.s. relative to $y$.

" $\Leftarrow$ " Since $\widetilde{A}$ is i.f.s. relative to $y$, that means $\forall x \in R^{n}, \lambda \in[0,1]$,

$$
\mu_{\tilde{A}}(\lambda(x-y)+y) \geq \mu_{\tilde{A}}(x)
$$

and 


$$
v_{\widetilde{A}}(\lambda(x-y)+y) \leq v_{\widetilde{A}}(x) .
$$

Take $\lambda=0$, we get $\mu_{\tilde{A}}(y) \geq \mu_{\tilde{A}}(x)$ and $v_{\tilde{A}}(y) \leq v_{\tilde{A}}(x)$ for all $x \in R^{n}$.

Thus,

$$
\begin{aligned}
\mu_{\tilde{A}}(\lambda(x-y)+y) & \geq \mu_{\tilde{A}}(x) \\
& \geq \min \left\{\mu_{\tilde{A}}(x), \mu_{\tilde{A}}(y)\right\} \\
v_{\tilde{A}}(\lambda(x-y)+y) & \leq v_{\widetilde{A}}(x) \\
& \leq \max \left\{v_{\tilde{A}}(x), v_{\widetilde{A}}(y)\right\}
\end{aligned}
$$

Hence, $\widetilde{A}$ is i.f.q-s. relative to $y$,

$$
\begin{aligned}
& \mu_{\tilde{A}}(y)=\sup _{x \in R^{n}}\left\{\mu_{\tilde{A}}(x)\right\}=1 \\
& v_{\widetilde{A}}(y)=\inf _{x \in R^{n}}\left\{v_{\widetilde{A}}(x)\right\}=0
\end{aligned}
$$

Theorem 3.4. Let $\tilde{A} \in F\left(R^{n}\right)$ is i.f.p-s. relative to $y \in R^{n}$, if

$$
\mu_{\tilde{A}}(y)=\sup _{x \in R^{n}}\left\{\mu_{\tilde{A}}(x)\right\}=1
$$

and

$$
v_{\widetilde{A}}(y)=\inf _{x \in R^{n}}\left\{v_{\widetilde{A}}(x)\right\}=0
$$

then it is i.f.s. relative to $y$.

Proof. It follows from Theorem 3.2, Theorem 3.3.

Remark 3.2. The inverse statements do not hold in general as shown in the following example.

Example 3.4. The intuitionistic fuzzy $\tilde{A} \in F\left(R^{n}\right)$ with

$$
\begin{aligned}
& \mu_{\tilde{A}}(x)= \begin{cases}\mathrm{e}^{x} & x \in(-\infty, 0] \\
\mathrm{e}^{-x} & x \in(0,+\infty)\end{cases} \\
& \nu_{\tilde{A}}(x)= \begin{cases}1-\mathrm{e}^{x} & x \in(-\infty, 0] \\
1-\mathrm{e}^{-x} & x \in(0,+\infty)\end{cases}
\end{aligned}
$$

is i.f.s. relative to $y=0$. But it is not i.f.p-s. relative to $y=0$.

\section{Basic Properties of Starshapedness of Intuitionistic Fuzzy Sets}

Proposition 4.1. If $\widetilde{A} \in F\left(R^{n}\right)$ is i.f.s. relative to $y \in R^{n}$, then

$$
\mu_{\tilde{A}}(y)=\sup _{x \in R^{n}}\left\{\mu_{\tilde{A}}(x)\right\}=1
$$

and

$$
v_{\tilde{A}}(y)=\inf _{x \in R^{n}}\left\{v_{\tilde{A}}(x)\right\}=0
$$

Proof. Let $\widetilde{A}$ is i.f.s. relative to $y$, then for all $x \in R^{n}, \lambda \in[0,1]$

$$
\mu_{\tilde{A}}(\lambda(x-y)+y) \geq \mu_{\tilde{A}}(x)
$$

and

$$
v_{\tilde{A}}(\lambda(x-y)+y) \leq v_{\tilde{A}}(x)
$$

Take $\lambda=0$, we get $\mu_{\tilde{A}}(y) \geq \mu_{\tilde{A}}(x)$ and $v_{\tilde{A}}(y) \leq v_{\tilde{A}}(x)$ for all $x \in R^{n}$.

So

$$
\mu_{\tilde{A}}(y)=\sup _{x \in R^{n}}\left\{\mu_{\tilde{A}}(x)\right\}=1
$$

and

$$
v_{\widetilde{A}}(y)=\inf _{x \in R^{n}}\left\{v_{\widetilde{A}}(x)\right\}=0
$$

Proposition 4.2. An intuitionistic fuzzy set $\widetilde{A} \in F\left(R^{n}\right)$ is i.f.s. relative to $y \in R^{n}$ iff for all $x \in R^{n}$, $\lambda \in[0,1]$, the following hold,

$$
\mu_{\tilde{A}}(\lambda x+y) \geq \mu_{\tilde{A}}(x+y)
$$

and

$$
v_{\tilde{A}}(\lambda x+y) \leq v_{\tilde{A}}(x+y)
$$

Proof. Supposed $A$ is i.f.s. relative to $y$, that is, for all $x \in R^{n}, \lambda \in[0,1]$,

$$
\mu_{\tilde{A}}(\lambda(x-y)+y) \geq \mu_{\tilde{A}}(x)
$$

and

$$
v_{\widetilde{A}}(\lambda(x-y)+y) \leq v_{\widetilde{A}}(x)
$$

Replacing $x$ by $x+y$ in the above inequality, we can get the desired result. Similarly, we can get the converse.

Proposition 4.3. An intuitionistic fuzzy set $\widetilde{A} \in F\left(R^{n}\right)$ is i.f.q-s. relative to $y \in R^{n}$ iff $\widetilde{A}_{[\alpha, \beta]}$ is starshaped set relative to $y$ for $\alpha \in\left[0, \mu_{\tilde{A}}(y)\right]$, $\beta \in\left[v_{\widetilde{A}}(y), 1\right]$.

Proof. " $\Rightarrow$ " Supposed $\widetilde{A}$ is i.f.q-s. relative to $y$, that is for all $x \in R^{n}, \lambda \in[0,1]$,

$$
\begin{aligned}
& \mu_{\tilde{A}}(\lambda(x-y)+y) \geq \min \left\{\mu_{\tilde{A}}(x), \mu_{\tilde{A}}(y)\right\} \\
& v_{\widetilde{A}}(\lambda(x-y)+y) \leq \max \left\{v_{\tilde{A}}(x), v_{\tilde{A}}(y)\right\}
\end{aligned}
$$

For any $\alpha \in\left[0, \mu_{\tilde{A}}(y)\right], \quad \beta \in\left[v_{\tilde{A}}(y), 1\right]$, if $x \in \widetilde{A}_{[\alpha, \beta]}$, then we have that $x, y \in \widetilde{A}_{[\alpha, \beta]}$. From the above inequality we get that

$$
\mu_{\tilde{A}}(\lambda(x-y)+y) \geq \alpha
$$


and

$$
v_{\widetilde{A}}(\lambda(x-y)+y) \leq \beta
$$

So $\overline{x y} \in \widetilde{A}_{[\alpha, \beta]}$.

“ $\Leftarrow$, For $x \in R^{n}, \lambda \in[0,1]$, if $\mu_{\widetilde{A}}(x)>\mu_{\widetilde{A}}(y)$, then let $\alpha=\mu_{\tilde{A}}(y)$. Accordingly we have $\overline{x y} \in \widetilde{A}_{[\alpha, \beta]}$, that is,

$$
\mu_{\widetilde{A}}(\lambda(x-y)+y) \geq \min \left\{\mu_{\widetilde{A}}(x), \mu_{\widetilde{A}}(y)\right\}
$$

If $\mu_{\widetilde{A}}(x) \leq \mu_{\widetilde{A}}(y)$, then let $\alpha=\mu_{\widetilde{A}}(x)$. Accordingly we have $\overline{x y} \in \widetilde{A}_{[\alpha, \beta]}$,that is,

$$
\mu_{\widetilde{A}}(\lambda(x-y)+y) \geq \min \left\{\mu_{\widetilde{A}}(x), \mu_{\tilde{A}}(y)\right\}
$$

If $v_{\widetilde{A}}(x)<v_{\widetilde{A}}(y)$, then let $\beta=v_{\widetilde{A}}(y)$.

Accordingly we have $\overline{x y} \in \widetilde{A}_{[\alpha, \beta]}$,that is,

$$
v_{\widetilde{A}}(\lambda(x-y)+y) \leq \max \left\{v_{\widetilde{A}}(x), v_{\widetilde{A}}(y)\right\}
$$

If $v_{\widetilde{A}}(x) \geq v_{\widetilde{A}}(y)$, then let $\beta=v_{\widetilde{A}}(x)$. Accordingly we have $x y \in \widetilde{A}_{[\alpha, \beta]}$, that is,

$$
v_{\widetilde{A}}(\lambda(x-y)+y) \leq \max \left\{v_{\widetilde{A}}(x), v_{\widetilde{A}}(y)\right\} .
$$

Thus $\widetilde{A}$ is i.f.q-s. relative to $y \in R^{n}$.

Proposition 4.4. An intuitionistic fuzzy set $\widetilde{A} \in F\left(R^{n}\right)$ is i.f.p-s. relative to $y$ iff $f . h p y\left(\mu_{\tilde{A}}\right)$ is starshaped relative to $\left(y, \mu_{\widetilde{A}}(y)\right)$ and $f . h p y\left(v_{\widetilde{A}}\right)$ is starshaped relative to $\left(y, v_{\tilde{A}}(y)\right)$.

Proof. “ $\Rightarrow$ ” If $\widetilde{A}$ is i.f.p-s. relative to $y$, $(x, t) \in f . h p y\left(\mu_{\tilde{A}}\right)$ and $(x, s) \in f . h p y\left(v_{\widetilde{A}}\right)$. Since $\widetilde{A}$ is i.f.p-s. relative to $y$ for any $\lambda \in[0,1]$ we have

$$
\begin{aligned}
\mu_{\tilde{A}}(\lambda(x-y)+y) & \geq \lambda \mu_{\widetilde{A}}(x)+(1-\lambda) \mu_{\tilde{A}}(y) \\
& \geq \lambda t+(1-\lambda) \mu_{\widetilde{A}}(y) \\
v_{\widetilde{A}}(\lambda(x-y)+y) & \leq \lambda v_{\widetilde{A}}(x)+(1-\lambda) v_{\tilde{A}}(y) \\
& \leq \lambda s+(1-\lambda) v_{\widetilde{A}}(y)
\end{aligned} .
$$

Thus, we have

$$
\begin{aligned}
& \lambda(x, t)+(1-\lambda)\left(y, \mu_{\widetilde{A}}(y)\right) \in f \cdot h y p\left(\mu_{\tilde{A}}\right) \\
& \lambda(x, s)+(1-\lambda)\left(y, v_{\widetilde{A}}(y)\right) \in f \cdot h y p\left(v_{\widetilde{A}}\right)
\end{aligned}
$$

Hence, $f$.hyp $\left(\mu_{\widetilde{A}}\right)$ is starshaped relative to $\left(y, \mu_{\widetilde{A}}(y)\right)$ and $f$.hyp $\left(v_{\widetilde{A}}\right)$ is starshaped relative to $\left(y, v_{\widetilde{A}}(y)\right)$.

“ $\Leftarrow$ Assume that $\left(x, \mu_{\tilde{A}}(x)\right) \in f . h p y\left(\mu_{\tilde{A}}\right)$ and $\left(x, v_{\widetilde{A}}(x)\right) \in f \cdot h p y\left(v_{\widetilde{A}}\right)$. By the starshapedness of $f \cdot h p y\left(\mu_{\tilde{A}}\right)$ and $f . h y p\left(v_{\widetilde{A}}\right)$ we can have

$$
\begin{aligned}
& \left(\lambda x+(1-\lambda)(y), \lambda \mu_{\tilde{A}}(x)+(1-\lambda) \mu_{\tilde{A}}(y)\right) \in f \cdot h p y\left(\mu_{\tilde{A}}\right) \\
& \left(\lambda x+(1-\lambda)(y), \lambda v_{\tilde{A}}(x)+(1-\lambda) v_{\tilde{A}}(y)\right) \in f \cdot h p y\left(v_{\tilde{A}}\right) .
\end{aligned}
$$

for any $\lambda \in[0,1]$. Thus $\widetilde{A}$ is i.f.p-s. relative to $y$.

A path in a set $S$ in $R^{n}$ is a continuous mapping $f:[0,1] \rightarrow S$. A set $S$ is said to be path connected if, there exists a path $f$ such that $f(0)=x$ and $f(1)=y$ for all $x, y \in S$ [12]. A intuitionistic fuzzy set $\widetilde{A}$ is said to be a path connected intuitionistic fuzzy set if its level sets are path connected [13]. Since a starshaped crisp set is path connected, one can easily prove the following proposition.

Proposition 4.5. If $\widetilde{A} \in F\left(R^{n}\right)$ is i.f.s. relative to $y \in R^{n}$, or is i.f.g.s., then $\widetilde{A}$ is a path connected intuitionistic fuzzy set.

Proof. It follows from Definition 3.1, Definition 3.2.

Proposition 4.6. If $\widetilde{A} \in F\left(R^{n}\right)$ is a intuitionistic fuzzy quasi-convex set, then it is i.f.g.s.. Furthermore, if $\widetilde{A} \in F\left(R^{n}\right)$ is i.f.g.s. then $\widetilde{A}$ is i.f.s., and is a fuzzy quasi-convex set.

Proof. If $\widetilde{A}$ is a intuitionistic fuzzy quasi-convex set, that for all $x, y \in R^{n}, \lambda \in[0,1]$, we have

$$
\begin{aligned}
& \mu_{\widetilde{A}}(\lambda(x-y)+y) \geq \min \left\{\mu_{\widetilde{A}}(x), \mu_{\widetilde{A}}(y)\right\} \\
& v_{\widetilde{A}}(\lambda(x-y)+y) \leq \max \left\{v_{\widetilde{A}}(x), v_{\widetilde{A}}(y)\right\}
\end{aligned}
$$

Then for all $x, y \in R^{n}$, the following hold,

$$
\begin{aligned}
& \mu_{\widetilde{A}}(\lambda(x-y)+y) \geq \min \left\{\mu_{\widetilde{A}}(x), \mu_{\widetilde{A}}(y)\right\} \geq \alpha \\
& v_{\widetilde{A}}(\lambda(x-y)+y) \leq \max \left\{v_{\widetilde{A}}(x), v_{\widetilde{A}}(y)\right\} \leq \beta
\end{aligned}
$$

So, $\overline{x y} \in \widetilde{A}_{[\alpha, \beta]}$. In other words $\widetilde{A}$ is i.f.g.s..

Additionally if $\widetilde{A} \in F\left(R^{n}\right)$ is i.f.g.s., then $\widetilde{A}_{[\alpha, \beta]}$ is starshaped. Thus they are path convex. In other words, they are intervals and there is at least one point $y$ in $\widetilde{A}_{[1,0]}$. It is well known that intervals are convex sets in $R$, so we have that $\widetilde{A}$ is i.f.s. relative to $\mathrm{y}$ and is a fuzzy quasi-convex set.

Proposition 4.7. If $\widetilde{A} \in F\left(R^{n}\right)$ is an intuitionistic fuzzy and the point $y \in R^{n}$ satisfies that $\mu_{\widetilde{A}}(y)=\inf _{x \in R^{n}}\left\{\mu_{\widetilde{A}}(x)\right\}$ and $v_{\widetilde{A}}(y)=\sup _{x \in R^{n}}\left\{v_{\widetilde{A}}(x)\right\}$. Then $\widetilde{A}$ is intuitionistic fuzzy quasi- starshaped set relative to $y$, that is, $y \in q-\operatorname{ker}(\widetilde{A})$. 
Proof. According to Definition 3.3, we have $\mu_{\tilde{A}}(y)=\inf _{x \in R^{n}}\left\{\mu_{\tilde{A}}(x)\right\}$ and $v_{\tilde{A}}(y)=\sup _{x \in R^{n}}\left\{v_{\tilde{A}}(x)\right\}$. So this statement is true.

Proposition 4.8. If $\widetilde{A}_{1}, \widetilde{A}_{2} \in F\left(R^{n}\right)$ are i.f.q-s. (respectively, i.f.p-s.) relative to $y \in R^{n}$. Then $\widetilde{A}_{1} \cap \widetilde{A}_{2}$ is i.f.q-s. (respectively, i.f.p-s.) relative to $y$.

Proof. Because that $\widetilde{A}_{1}, \widetilde{A}_{2} \in F\left(R^{n}\right)$ are i.f.q-s. relative to $y \in R^{n}$, for all $x \in R^{n}, \lambda \in[0,1]$, we have

$$
\begin{gathered}
\mu_{\widetilde{A}_{i}}(\lambda x+(1-\lambda) y) \geq \min \left\{\mu_{\widetilde{A}_{i}}(x), \mu_{\widetilde{A}_{i}}(y)\right\} \\
v_{\widetilde{A}_{i}}(\lambda x+(1-\lambda) y) \leq \max \left\{v_{\widetilde{A}_{i}}(x), v_{\widetilde{A}_{i}}(y)\right\}, i=1,2
\end{gathered}
$$

So,

$$
\begin{aligned}
& \left(\mu_{\widetilde{A}_{1}} \cap \mu_{\widetilde{A}_{2}}\right)(\lambda x+(1-\lambda) y) \\
& =\max \left\{\mu_{\widetilde{A}_{i}}(\lambda x+(1-\lambda) y), i=1,2\right\} \\
& \geq \min \left\{\max \left\{\mu_{\widetilde{A}_{1}}(x), \mu_{2}(x)\right\}, \mu_{\widetilde{A}_{2}}(y)\right\} \\
& =\min \left\{\left(\mu_{\widetilde{A}_{1}} \cup \mu_{\widetilde{A}_{2}}\right)(x),\left(\mu_{\widetilde{A}_{1}} \cup \mu_{\widetilde{A}_{2}}\right)(y)\right\}
\end{aligned}
$$

and,

$$
\begin{aligned}
& \left(v_{\widetilde{A}_{1}} \cup v_{\widetilde{A}_{2}}\right)(\lambda x+(1-\lambda) y) \\
& =\max \left\{v_{\widetilde{A}_{i}}(\lambda x+(1-\lambda) y), i=1,2\right\} \\
& \leq \max \left\{\max \left\{v_{\widetilde{A}_{1}}(x), v_{\widetilde{A}_{1}}(y)\right\}, \max \left\{v_{\widetilde{A}_{2}}(x), v_{\widetilde{A}_{2}}(y)\right\}\right\} \\
& =\max \left\{\left(v_{\widetilde{A}_{1}} \cup v_{\widetilde{A}_{2}}\right)(x),\left(v_{\widetilde{A}_{1}} \cup v_{\widetilde{A}_{2}}\right)(y)\right\}
\end{aligned}
$$

Hence, $\widetilde{A}_{1} \cap \widetilde{A}_{2}$ is i.f.q-s. relative to $y$.

If $\widetilde{A}_{1}, \widetilde{A}_{2} \in F\left(R^{n}\right)$ are i.f.p-s. relative to $y \in R^{n}$. Then all $x \in R^{n}, \lambda \in[0,1]$, we have

$$
\begin{gathered}
\mu_{\widetilde{A}_{i}}(\lambda(x-y)+y) \geq \lambda \mu_{\widetilde{A}_{i}}(x)+(1-\lambda) \mu_{\widetilde{A}_{i}}(y), \\
v_{\widetilde{A}_{i}}(\lambda(x-y)+y) \leq \lambda v_{\widetilde{A}_{i}}(x)+(1-\lambda) v_{\widetilde{A}_{i}}(y), \quad i=1,2
\end{gathered}
$$

So,

$$
\begin{aligned}
& \left(\mu_{\widetilde{A}_{1}} \cap \mu_{\widetilde{A}_{2}}\right)(\lambda x+(1-\lambda) y) \\
& =\min \left\{\mu_{\widetilde{A}_{i}}(\lambda x+(1-\lambda) y), i=1,2\right\} \\
& \geq \min \left\{\lambda \mu_{\widetilde{A}_{i}}(x)+(1-\lambda) \mu_{\widetilde{A}_{i}}(y), i=1,2\right\} \\
& \geq \lambda \min \left\{\mu_{\widetilde{A}_{1}}(x), \mu_{\widetilde{A}_{2}}(x)\right\}+(1-\lambda) \min \left\{\mu_{\widetilde{A}_{1}}(y), \mu_{\widetilde{A}_{2}}(y)\right\} \\
& =\lambda\left(\mu_{\widetilde{A}_{1}} \cap \mu_{\widetilde{A}_{2}}\right)(x)+(1-\lambda)\left(\mu_{\widetilde{A}_{1}} \cap \mu_{\widetilde{A}_{2}}\right)(y)
\end{aligned}
$$

and

$$
\begin{aligned}
& \left(v_{\widetilde{A}_{1}} \cup v_{\widetilde{A}_{2}}\right)(\lambda x+(1-\lambda) y) \\
& =\max \left\{v_{\widetilde{A}_{i}}(\lambda x+(1-\lambda) y), i=1,2\right\} \\
& \leq \max \left\{\lambda v_{\widetilde{A}_{i}}(x)+(1-\lambda) v_{\widetilde{A}_{i}}(y), i=1,2\right\} \\
& \leq \lambda \max \left\{v_{\widetilde{A}_{1}}(x), v_{\widetilde{A}_{2}}(x)\right\}+(1-\lambda) \max \left\{v_{\widetilde{A}_{1}}(y), v_{\widetilde{A}_{2}}(y)\right\} \\
& =\lambda\left(v_{\widetilde{A}_{1}} \cap v_{\widetilde{A}_{2}}\right)(x)+(1-\lambda)\left(v_{\widetilde{A}_{1}} \cap v_{\widetilde{A}_{2}}\right)(y)
\end{aligned}
$$

Hence $\widetilde{A}_{1} \cap \widetilde{A}_{2}$ is i.f.p-s. relative to $y$.

Proposition 4.9. If $\widetilde{A}_{1}, \widetilde{A}_{2} \in F\left(R^{n}\right)$ are i.f.q-s. (respectively, i.f.p-s.) relative to $y \in R^{n}$ and $\mu_{\widetilde{A}_{1}}(y)=\mu_{\widetilde{A}_{2}}(y), \quad v_{\widetilde{A}_{1}}(y)=v_{\widetilde{A}_{2}}(y)$. Then $\widetilde{A}_{1} \cup \widetilde{A}_{2}$ is i.f.q-s. (respectively, i.f.p-s.) relative to $y$.

Proof. Since $\widetilde{A}_{1}, \widetilde{A}_{2} \in F\left(R^{n}\right)$ are i.f.q-s. relative to $y \in R^{n}$, for all $x \in R^{n}, \lambda \in[0,1]$, we have

$$
\mu_{\widetilde{A}_{i}}(\lambda x+(1-\lambda) y) \geq \min \left\{\mu_{\widetilde{A}_{i}}(x), \mu_{\widetilde{A}_{i}}(y)\right\}
$$

and

$$
v_{\widetilde{A}_{i}}(\lambda x+(1-\lambda) y) \leq \max \left\{v_{\widetilde{A}_{i}}(x), v_{\widetilde{A}_{i}}(y)\right\}, i=1,2
$$
get

By $\mu_{\widetilde{A}_{1}}(y)=\mu_{\widetilde{A}_{2}}(y)$ and $v_{\widetilde{A}_{1}}(y)=v_{\widetilde{A}_{2}}(y)$, we can

$$
\begin{aligned}
& \left(\mu_{\widetilde{A}_{1}} \cup \mu_{\widetilde{A}_{2}}\right)(\lambda x+(1-\lambda) y) \\
& =\max \left\{\mu_{\widetilde{A}_{i}}(\lambda x+(1-\lambda) y), i=1,2\right\} \\
& \geq \min \left\{\max \left\{\mu_{\widetilde{A}_{1}}(x), \mu_{2}(x)\right\}, \mu_{\widetilde{A}_{2}}(y)\right\} \\
& =\min \left\{\left(\mu_{\widetilde{A}_{1}} \cup \mu_{\widetilde{A}_{2}}\right)(x),\left(\mu_{\widetilde{A}_{1}} \cup \mu_{\widetilde{A}_{2}}\right)(y)\right\}
\end{aligned}
$$

and

$$
\begin{aligned}
& \left(v_{\widetilde{A}_{1}} \cap v_{\widetilde{A}_{2}}\right)(\lambda x+(1-\lambda) y) \\
& =\min \left\{v_{\widetilde{A}_{i}}(\lambda x+(1-\lambda) y), i=1,2\right\} \\
& \leq \max \left\{\min \left\{v_{\widetilde{A}_{1}}(x), v_{\widetilde{A}_{2}}(x)\right\}, v_{\widetilde{A}_{1}}(y)\right\} \\
& =\max \left\{\left(v_{\widetilde{A}_{1}} \cap v_{2}\right)(x),\left(v_{\widetilde{A}_{1}} \cap v_{\widetilde{A}_{2}}\right)(y)\right\}
\end{aligned}
$$

Hence $\widetilde{A}_{1} \cup \widetilde{A}_{2}$ is i.f.q-s. relative to $y$.

Since $\widetilde{A}_{1}, \widetilde{A}_{2} \in F\left(R^{n}\right)$ are i.f.p-s. relative to $y \in R^{n}$, for all $x \in R^{n}, \lambda \in[0,1]$, we have

$$
\mu_{\widetilde{A}_{i}}(\lambda x+(1-\lambda) y) \geq \lambda \mu_{\tilde{A}_{i}}(x)+(1-\lambda) \mu_{\widetilde{A}_{i}}(y)
$$

and

$$
v_{\widetilde{A}_{i}}(\lambda(x-y)+y) \leq \lambda v_{\widetilde{A}_{i}}(x)+(1-\lambda) v_{\widetilde{A}_{i}}(y), \quad i=1,2
$$


From $\mu_{\widetilde{A}_{1}}(y)=\mu_{\tilde{A}_{2}}(y)$ and $v_{\widetilde{A}_{1}}(y)=v_{\widetilde{A}_{2}}(y)$ we can find

$$
\begin{aligned}
& \left(\mu_{\widetilde{A}_{1}} \cup \mu_{\widetilde{A}_{2}}\right)(\lambda x+(1-\lambda) y) \\
& =\max \left\{\mu_{\widetilde{A}_{i}}(\lambda x+(1-\lambda) y), i=1,2\right\} \\
& \geq \max \left\{\lambda \mu_{\tilde{A}_{i}}(x)+(1-\lambda) \mu_{\tilde{A}_{i}}(y), i=1,2\right\} \\
& =\lambda\left(\mu_{\widetilde{A}_{1}} \cup \mu_{\widetilde{A}_{2}}\right)(x)+(1-\lambda)\left(\mu_{\widetilde{A}_{1}} \cup \mu_{\widetilde{A}_{2}}\right)(y)
\end{aligned}
$$

and

$$
\begin{aligned}
& \left(v_{\widetilde{A}_{1}} \cap v_{2}\right)(\lambda x+(1-\lambda) y) \\
& =\min \left\{v_{\widetilde{A}_{i}}(\lambda x+(1-\lambda) y), i=1,2\right\} \\
& \leq \min \left\{\lambda v_{\widetilde{A}_{i}}(x)+(1-\lambda) v_{\widetilde{A}_{i}}(y), i=1,2\right\} \\
& =\lambda\left(v_{\widetilde{A}_{1}} \cap v_{\widetilde{A}_{2}}\right)(x)+(1-\lambda)\left(v_{\widetilde{A}_{1}} \cap v_{\widetilde{A}_{2}}\right)(y)
\end{aligned}
$$

Hence $\widetilde{A}_{1} \cup \widetilde{A}_{2}$ is i.f.p-s. relative to $y$.

Let $x_{0} \in R^{n}, \widetilde{A} \in F\left(R^{n}\right)$, then the translation of $\widetilde{A}$ by $x_{0}$ is the intuitionistic fuzzy $x_{0}+\tilde{A}$ defined as $\left(x_{0}+\tilde{A}\right)(x)=\widetilde{A}\left(x-x_{0}\right)$.

Proposition 4.10. If $\widetilde{A} \in F\left(R^{n}\right)$ is i.f.s. (respectively, i.f.p-s., i.f.q-s.) relative to $y$ and $x_{0} \in R^{n}$. Then $x_{0}+\widetilde{A}$ is i.f.s. (respectively, i.f.p-s., i.f.q-s.) relative to $x_{0}+y$.

Proof. We only give the proof for the case of intuitionistic fuzzy starshapedness. Similarly, the others can be proved.

For any $x \in R^{n}$, since $\widetilde{A} \in F\left(R^{n}\right)$ is i.f.s. relative to $y$. We have that

$$
\begin{aligned}
& \left(x_{0}+\mu_{\tilde{A}}\right)\left(\lambda\left(x-y-x_{0}\right)+y+x_{0}\right) \\
& =\mu_{\tilde{A}}\left(\lambda\left(x-y-x_{0}\right)+y\right) \geq \mu_{\tilde{A}}\left(x-x_{0}\right) \\
& =\left(x_{0}+\mu_{\tilde{A}}\right)(x)
\end{aligned}
$$

and

$$
\begin{aligned}
& \left(x_{0}+v_{\tilde{A}}\right)\left(\lambda\left(x-y-x_{0}\right)+y+x_{0}\right) \\
& =v_{\widetilde{A}}\left(\lambda\left(x-y-x_{0}\right)+y\right) \leq v_{\tilde{A}}\left(x-x_{0}\right) \\
& =\left(x_{0}+v_{\widetilde{A}}\right)(x)
\end{aligned}
$$

So, $x_{0}+\widetilde{A}$ is i.f.s. relative to $x_{0}+y$.

Let $T$ be a linear invertible transformation on $R^{n}$, $\widetilde{A} \in F\left(R^{n}\right)$. Then by the Extension Principle we have that $(T(\widetilde{A}))(x)=\widetilde{A}\left(T^{-1}(x)\right)$.

Proposition 4.11 If $\widetilde{A} \in F\left(R^{n}\right)$ is i.f.s. (respectively, i.f.p-s., i.f.q-s.) relative to $y$ and $T$ is a linear invertible transformation on $R^{n}$. Then $T(\widetilde{A})$ is i.f.s. (respectively, i.f.p-s., i.f.q-s.) relative to $T(y)$.

Proof. We only give the proof for the case of intuitionistic fuzzy quasi-starshapedness. Similarly, the others can be proved.

For any $x \in R^{n}$, since $\tilde{A} \in F\left(R^{n}\right)$ is i.f.q-s. relative to $y$. We have that

$$
\begin{aligned}
(T(\widetilde{A}))(\lambda x+(1-\lambda) T(y)) & =A\left(\lambda T^{-1}(x)+(1-\lambda) y\right) \\
& \geq \min \left\{\widetilde{A}\left(T^{-1}(x)\right), \widetilde{A}(y)\right\} \\
& =\min \{(T \widetilde{A}(x)), T(\widetilde{A})(T(y))\}
\end{aligned}
$$

Hence, $T(\widetilde{A})$ is i.f.q-s. relative to $T(y)$.

\section{Conclusions}

Intuitionistic fuzzy set and fuzzy starshaped set are some special fuzzy sets. In this article, we introduce some new different types of intuitionistic fuzzy starshaped set. By discussing the relationships among these different types of starshapedness, and obtained some important properties. Deepening people's understanding of intuitionistic fuzzy sets, enrich and perfect the theories of fuzzy sets.

\section{Acknowledgements}

The project is supported by the postdoctoral Science Foundation of China (NO.20100481331) and the National Natural Science Foundation of China (NO. 71071124, 11001227).

\section{References}

[1] K. Atanassov, "Intuitionistic Fuzzy Sets,” Fuzzy Sets and Systems, Vol. 20, No. 1, 1986, pp. 87-96. doi:10.1016/S0165-0114(86)80034-3

[2] S. Manro, "Common Fixed Point Theorems in Intuitionistic Fuzzy Metric Spaces,” Applied Mathematics, No. 1, 2010, pp. 510-514. doi:10.4236/am.2010.16067

[3] G. Deschrijive and E. E. Kerre, "On the Position of Intuitionistic Fuzzy Set Theory in the Framework of Theories Modelling Imprecision,” Information Sciences, Vol. 177, No. 8, 2007, pp. 1860-1866.

doi:10.1016/j.ins.2006.11.005

[4] L. Lin, X. H. Yuan and Z. Q. Xia, "Multicriteria Fuzzy Decision-Making Methods Based on Intuitionistic Fuzzy Sets,” Journal of Computer and System Science, Vol. 73, No. 1, 2007, pp. 84-88. doi:10.1016/j.jcss.2006.03.004

[5] L. K. Vlachos and G. D. Sergiadis, "Intuitionistic Fuzzy 
Information-Applications to Pattern Recognition,” Pattern Recognition Letters, Vol. 28, No. 2, 2007, pp. 197206. doi:10.1016/j.patrec.2006.07.004

[6] H. Bustince and P. Burillo, "Structures on Intuitionistic Fuzzy Relations,” Fuzzy Sets and Systems, Vol. 78, No. 3, 1996, pp. 293-303.

doi:10.1016/0165-0114(96)84610-0

[7] M. D. Cock, C. Cornelis and E. E. Kerre, "Intuitionistic Fuzzy Relational Images," Studies in Computational Intelligence, Vol. 2, 2005, pp. 129-145.

[8] D. Coker, “An Introduction to Intuitionistic Fuzzy Topological Spaces,” Fuzzy Sets and Systems, Vol. 88, No. 1, 1997, pp. 81-89. doi:10.1016/S0165-0114(96)00076-0

[9] G. Deschrijive and E. E. Kerre, "On the Composition of
Intuitionistic Fuzzy Relations,” Fuzzy Sets and Systems, Vol. 136, No. 3, 2003, pp. 333-361.

doi:10.1016/S0165-0114(02)00269-5

[10] D. Qiu, L. Shu and Zh. W. Mo, “On Starshaped Fuzzy Sets,” Fuzzy Sets and Systems, Vol. 160, No. 11, 2009, pp. 1563-1577. doi:10.1016/j.fss.2008.11.005

[11] M. Li, “Cut Sets of Intuitionistic Fuzzy Sets," Journal of Liaoning Normal University Vol. 30, No. 2, 2007, pp. 152-154.

[12] Y. R. Syau, “Closed and Convex Fuzzy Sets,” Fuzzy Sets and Systems, Vol. 110, No. 2, 2000, pp. 287-291. doi:10.1016/S0165-0114(98)00082-7

[13] J. G. Brown, “A Note on Fuzzy Set,” Information and Control, Vol. 18, No. 1, 1971, pp. 32-39. doi:10.1016/S0019-9958(71)90288-9 